\title{
NUMP E SILENT NOUNS: FRONTEIRAS SINTÁTICAS NA MARCAÇÃO DE PLURAL NO PB ${ }^{1}$
}

\section{NUMP AND SILENT NOUNS: SYNTACTIC BOUNDARIES FOR PLURAL MARKING IN BP}

\author{
Bruna Karla Pereira ${ }^{2}$ \\ Universidade Federal dos Vales do Jequitinhonha e Mucuri \\ Diamantina, Minas Gerais, Brasil
}

RESUMO: Neste artigo, investiga-se a distribuição do morfema de plural, na estrutura interna do DP, em português brasileiro (PB) não padrão. Argumenta-se que essa distribuição é determinada pela posição de cardinais (DANON, 2011; NORRIS, 2014) ou de silent nouns (KAYNE, 2005). Assim, propõe-se que: (I) a posição dos cardinais divide o DP em dois domínios nos quais sintagmas à esquerda de NumP são marcados com o morfema '-s' de plural, enquanto sintagmas à direita de NumP não são; (II) a posição de silent nouns exerce basicamente essa mesma função, em estruturas nas quais os cardinais não podem aparecer; e (III) os traços de número são valorados e interpretáveis em Num (AUGUSTO et al., 2006) e se tornam valorados em D e N após checagem via concordância (PESETSKY; TORREGO, 2007).

PALAVRAS-CHAVE: Concordância nominal em PB; Morfema de plural; NumP; Silent Nouns.

ABSTRACT: This paper examines the DP-internal distribution of the plural morpheme in non-standard Brazilian Portuguese (BP) and argues that this is determined by the position of either cardinals (DANON,

\footnotetext{
${ }^{1}$ Este artigo é uma versão traduzida e resumida do texto publicado no MIT Working Papers in Linguistics, que pode ser obtido em $<$ http://mitwpl.mit.edu/catalog/mwpl81/>, conforme referência seguinte: PEREIRA, Bruna Karla. The DP-internal distribution of the plural morpheme in Brazilian Portuguese. MIT Working Papers in Linguistics (Papers on Morphology, edited by Snejana Iovtcheva and Benjamin Storme), v. 81, p. 85-104, 2017.

${ }^{2}$ Agradecimentos: Esta pesquisa recebeu o apoio da CAPES (Coordenação de Aperfeiçoamento de Pessoal de Nível Superior, Ministério da Educação, Brasília - DF, CEP: 70.040-020), sob registro $n^{\circ}$. 0751/2015-04 no programa de Pesquisa Pós-Doutoral no Exterior, e foi conduzida no MIT (Massachusetts Institute of Technology, Cambridge, Massachusetts, USA), com vínculo de visiting scholar.
} 
2011; NORRIS, 2014) or silent nouns (KAYNE, 2005). Therefore, I propose that: (I) cardinals divide BP DPs into two domains in which phrases that precede NumP are marked with the plural morpheme while phrases that follow it are unmarked; (II) silent nouns have basically the same function in structures where cardinals cannot appear; and (III) number features are interpretable and valued in Num (AUGUSTO et al., 2006) and become valued in $\mathrm{D}$ and $\mathrm{N}$ after agreement (PESETSKY; TORREGO, 2006).

KEYWORDS: Brazilian Portuguese nominal concord; Plural morpheme; NumP; Silent nouns. 


\section{INTRODUÇÃO}

Neste trabalho, analiso a distribuição do morfema de plural em português do Brasil (PB) não padrão (1a, b). Diferentemente do PB padrão (1c), que apresenta todos os elementos do DP marcados com o morfema '-s' de plural, o PB não padrão $(1 \mathrm{a}, \mathrm{b})$ apresenta a maior parte deles não marcada.
a. Os outro carro branco
b. Os outros carro branco
c. Os outros carros brancos

Considerando esses fatos, investigarei as condições sintáticas que determinam quais sintagmas devem, podem ou não podem receber marca de plural em PB não padrão. Para isso, assumirei que a posição do numeral cardinal no DP funciona como uma fronteira dividindo DPs do PB em dois domínios, sendo que sintagmas à esquerda dessa posição são marcados com o morfema de plural, enquanto sintagmas à sua direita não são. Como observado, 'os', em (1a, d), e 'os' mais 'outros', em ( $1 \mathrm{~b}, \mathrm{e})$, precedem o cardinal e recebem marca de plural, enquanto os itens que sucedem o cardinal não recebem essa marca.

d. Os dois outro carro branco

e. Os outros dois carro branco

Com vistas a demonstrar essa investigação, o artigo está organizado em três seções principais. A seção 2 apresenta, inicialmente, propostas vigentes (COSTA; FIGUEIREDO SILVA, 2006); posteriormente, a base teórica (PESETSKY; TORREGO, 2007) utilizada para se assumir uma nova proposta; e, finalmente, dados de outras línguas para ilustrar que cardinais funcionam como uma fronteira sintática dividindo o DP em duas fases no que se refere à distribuição de traços de número no interior de sua estrutura (DANON, 2011; NORRIS, 2014). Por sua vez, a seção 3 testa, com dados do PB, o poder explanatório dessa análise e acrescenta que silent nouns (KAYNE, 2005) desempenham o mesmo papel de fronteira sintática, em estruturas nominais que apresentam restrições à ocorrência de cardinais. Por último, a seção 4 descreve estruturas de PB dialetal com determinantes $W h$ e cardinais, que oferecem suporte para a análise proposta.

\section{BASE TEÓRICA}

Esta seção é dividida em quatro outras subseções: a primeira (2.1) discute propostas vigentes para o problema estudado; a segunda (2.2) apresenta a base teórica para se desenvolver uma nova proposta; a terceira (2.3) fornece exemplos de línguas nas quais o numeral cardinal se configura como uma fronteira na distribuição de traços de número no DP; e a quarta (2.4) mostra exemplos de 
línguas nas quais categorias funcionais nulas (silent nouns) também desempenham essa função.

\subsection{Propostas vigentes}

Costa e Figueiredo Silva (2006) assumem que o morfema de plural é singleton no PB e dissociado no português europeu (PE). Devido a essa diferença, nessa proposta, o PB permite a presença do morfema de plural em um único elemento do DP, normalmente D, como visto em (1a), enquanto o PE permite que o morfema "se espalhe em todos os outros elementos capazes de recebê-lo" (COSTA; FIGUEIREDO SILVA, 2006, p. 38, tradução minha), como visto em (1c) acima.

Com efeito, é fato que, em PB não padrão, o morfema de plural pode aparecer no determinante, mas também é verdade que ele pode aparecer em mais de um elemento do DP: por exemplo, no determinante e no adjetivo (1b) ${ }^{4}$. Essa é a razão por que Castro e Pratas (2006, p. 18) assumem que:

In most cases the plural marker seems to surface as a singleton, but in others the plural is marked in two different positions [...] C\&FS [COSTA; FIGUEIREDO SILVA, 2006] do not account for the 'half-way' singleton morpheme [...] these patterns must be subject to further investigation.

Em suma, o fato de que o morfema de plural pode aparecer em mais de um elemento do DP evidencia que ele não é singleton, contra o que é assumido por Costa e Figueiredo Silva (2006). Ainda, nessa proposta, os autores admitem que $\mathrm{D}$ é o núcleo nominal ligado à LF (Logical Form) para interpretação de número (COSTA; FIGUEIREDO SILVA, 2006, p. 37), sem discussão a respeito do papel desempenhado por Num nesta questão. Sobretudo, essa proposta não explica as restrições sintáticas que determinam a distribuição do morfema de plural no domínio do DP em PB não padrão, visto que se pauta na ideia de autonomia do componente morfológico 5 .

\subsection{Em direção a uma nova proposta}

O termo 'traço-phi' é utilizado para abarcar categorias que envolvem concordância (como pessoa, gênero e número no DP) e que são analisadas sob os conceitos de valoração e interpretabilidade.

\footnotetext{
3 "to spread over all elements able to bear it" (COSTA; FIGUEIREDO SILVA, 2006, p. 38).

${ }^{4} \mathrm{O}$ morfema de plural pode aparecer em mais de duas posições, como em uma sequência com determinante, possessivo e adjetivo precedendo NumP: "Os meus outros (dois) livro pequeno".

5 "autonomous morphological component [...] partly independent from syntax" (COSTA; FIGUEIREDO SILVA, 2006, p. 44).
} 
Quanto ao conceito de valoração, de acordo com Pesetsky e Torrego (2007, p. 263, tradução minha), "Certos traços, em itens lexicais, parecem vir não valorados do léxico e recebem seu valor de uma instância valorada com os mesmos traços, presentes em um outro item lexical"6 (PESETSKY; TORREGO, 2007, p. 263). Por exemplo, gênero é uma propriedade nominal e vem do léxico valorado no nome $(\mathrm{N})$. Em contraste, em $\mathrm{D}$ e $\mathrm{A}$, o traço de gênero vem do léxico não valorado e se "torna valorado como consequência de um processo de concordância com o traço de gênero de N"7 (PESETSKY; TORREGO, 2007, p. 263, tradução minha). Em $\mathrm{D}$ e $\mathrm{A}$, também o número é lexicalmente não valorado, mas se torna "valorado como resultado de concordância com N" (PESETSKY; TORREGO, 2007, p. 263, tradução minha).

Quanto ao conceito de interpretabilidade, a distinção entre interpretável e não interpretável está relacionada a "se um traço de um item lexical particular promove ou não uma contribuição semântica para a interpretação daquele item"" (PESETSKY; TORREGO, 2007, p. 264, tradução minha). Por exemplo, "os traços de número e pessoa, no DP, podem promover uma contribuição crucial para a interpretação semântica"10 (PESETSKY; TORREGO, 2007, p. 264, tradução minha), mas os traços de número, em $\mathrm{A}$, não parecem ter contribuição para o significado.

Tendo feito esse breve resumo sobre os conceitos de valoração e interpretabilidade, é importante perguntar como é possível identificar se o traço de número no nome é lexicalmente valorado e também como a concordância em número é desencadeada no DP.

Para a primeira questão, uma explicação, de acordo com Pesetsky e Torrego (2007), está relacionada a nomes pluralia tantum, como '(these) scissors'. A existência desses nomes no inglês indica que $\mathrm{N}$ vem do léxico com o traço de número valorado; ao contrário, a inexistência de $\mathrm{D}$ ou A pluralia tantum indica que esses itens vêm do léxico com os traços de número não valorados.

Entretanto, nem todas as línguas têm nomes pluralia tantum. De acordo com Pesetsky e Torrego (2007), no espanhol, por exemplo, nomes pluralia tantum propriamente ditos parecem não existir, o que desencadeia um entendimento diferente da fonte de traços de número nessa língua. Baseados em estudos prévios, Pesetsky e Torrego (2007) sugerem que a categoria de número no espanhol é um traço de NumP. De modo semelhante, Blühdorn et al. (2008)

\footnotetext{
6 "Certain features on lexical items appear to come from the lexicon unvalued, and receive their value from a valued instance of the same feature, present on another lexical item" (PESETSKY; TORREGO, 2007, p. 263).

7 "valued as a consequence of a syntactic process of agreement with the gender feature of N" (PESETSKY; TORREGO, 2007, p. 263).

8 “valued as a result of agreement with N" (PESETSKY; TORREGO, 2007, p. 263).

9 "whether or not a feature of a particular lexical item makes a semantic contribution to the interpretation of that item" (PESETSKY; TORREGO, 2007, p. 264).

10 "the person and number features on DP may make a crucial contribution to semantic interpretation. The corresponding features on $\mathrm{V}$ appear to make no contribution [...] Likewise for the number features of A.” (PESETSKY; TORREGO, 2007, p. 264-5).
} 
assumem que o PB não dispõe de pluralia tantum propriamente ditos, ${ }^{11} \mathrm{o}$ que significa que os traços de número nos nomes $(\mathrm{N})$ são lexicalmente não valorados. Portanto, a partir dessas observações e dos argumentos apresentados por Augusto et al. (2006), assumo, com esses autores, que o locus de número no $\mathrm{PB}$ não é $\mathrm{N}$ nem D, mas Num, como no espanhol.

Para a segunda questão, de acordo com Chomsky (2001), a concordância ocorre quando uma sonda com traços não interpretáveis busca seu alvo com traços interpretáveis a fim de se tornar valorada. Uma vez que traços não interpretáveis são valorados, eles devem ser apagados. Em uma versão reformulada dessa proposta, uma das consequências de se adotar uma abordagem de traços compartilhados (feature sharing), como defendida por Pesetsky e Torrego (2007), é que, depois de a valoração ocorrer, o traço não é apagado, mas permanece disponível para outra sonda.

Uma outra consequência é a independência entre valoração e interpretabilidade. Assim, espera-se que o léxico tenha quatro tipos de traços, como se segue:

Tabela 1: Tipos de traços

\begin{tabular}{|l|l|l|l|}
\hline$u \mathrm{~F}$ val & não interpretável, valorado & $i \mathrm{~F}$ val & interpretável, valorado \\
\hline$u \mathrm{~F}[\mathrm{]}]$ & não interpretável, não valorado & $i \mathrm{~F}[\mathrm{]}]$ & interpretável, não valorado \\
\hline
\end{tabular}

Fonte: (PESETSKY; TORREGO, 2007, p. 269, tradução minha)

Em suma, D e A sondam NumP (em algumas línguas, e NP em outras) como alvo para valoração de traços de número. A seção 3 mostrará como esse mecanismo se aplica ao PB. Entretanto, antes de chegar à análise dos dados, algumas questões teóricas referentes à relação entre a posição de cardinais e a distribuição dos traços de número no DP serão abordadas.

\subsection{NumP: fronteira sintática na distribuição dos traços de número no DP}

Conforme observado por Danon (2011, p. 301), em muitas línguas, a distribuição dos traços de número plural é determinada pela posição dos cardinais na estrutura do DP. Por exemplo, no finlandês (2), "um traço de número (plural) está disponível somente acima da posição do numeral" ${ }^{12}$ (DANON, 2011, p. 302, tradução minha). Além disso, Norris (2014) apresenta exemplos do estoniano (3)

\footnotetext{
${ }^{11}$ No PB, a palavra 'óculos', ao contrário do que preveem as gramáticas tradicionais, é frequentemente usada com modificadores no singular. A maior parte dos falantes entende que 'o óculos' se refere a um único objeto, enquanto 'os óculos' se refere a mais de um objeto. Por isso, não se trata de um nome pluralia tantum propriamente.

12 "a (plural) number feature is only available above the position of the numeral" (DANON, 2011, p. 302).
} 
em que "material à esquerda do numeral é plural, e material à direita é singular"13 (NORRIS, 2014, p. 143, tradução minha).

"Ne kaksi pien-tä auto-a seiso-ivat tiellä. those.PL two.SG small-PART.SG car-PART.SG stand-PAST.3PL road.ADESS 'Those two small cars stood at the road' (BRATTICO, 2010)"

(DANON, 2011, p. 301).

$$
\begin{array}{ll}
\text { “nee-d } & \text { viis ilusa-t maja } \\
\text { this-PL.NOM } & \text { 5.NOM beautiful-PAR house.PAR } \\
\text { 'these five beautiful houses' (ERELT et al. 1993b, p. 143)" }
\end{array}
$$

(NORRIS, 2014, p. 144).

Em resumo, as línguas do mundo diferem no que diz respeito à marcação de plural no domínio interno ao DP. Em muitas delas, a distribuição dos traços de número é determinada pela posição do numeral cardinal na estrutura do DP.

\subsection{Categorias vazias e a distribuição dos traços de número no DP}

Kayne (2005, p. 241-242, tradução minha) ${ }^{14}$ observa que adjetivos funcionais, como $f e w(4 \mathrm{~b})$, "modificam um nome distinto do nome visível 'books' no plural [...] O nome em questão é a contraparte silenciosa da palavra 'number' vista em:"15 (4c).
a. "*a books"
b. "a few books"
c. "a small number of books"

Assim sendo, em contraste com (4a), que é agramatical porque o artigo indefinido ' $a$ ' do inglês não é compatível com nomes no plural, (4b) é gramatical porque 'few' modifica a palavra NUMBER no singular, que não é realizada foneticamente e que é seguida de uma preposição 'of', como visto em (4c). Essa hipótese se estende, entre outros, a 'much', que modifica uma categoria nula do tipo AMOUNT, bem como a 'clock/time', que modifica uma categoria nula do tipo HOUR. Essas categorias funcionais vazias são os silent nouns.

Além disso, traços de número em silent nouns podem variar de uma língua para outra. Por exemplo, no italiano (5a) e no francês (5b), um artigo definido no

\footnotetext{
13 "material to the left of the numeral is plural, and material to the right is singular" (NORRIS, 2014, p. 143).

${ }^{14}$ Agradeço a Alexandru Nicolae (University of Bucharest) por ter despertado minha atenção para a proposta referente aos silent nouns.

15 "modify a noun distinct from the visible plural 'books' [...] The noun in question is a silent counterpart of the overt 'number' seen in:" (KAYNE, 2005, p. 241-242).
} 
plural pode coocorrer com sintagmas no singular, o que indica que, nessas línguas, o silent noun HOUR é plural.

(5) a. "Sono le ore una.

('are the(pl.) hours one')" (KAYNE, 2005, p. 259).

'It's one o'clock'.

b. "Vers les ${ }^{16}$ une heure.

('toward the(pl.) one hour' =

'around one o'clock')" (KAYNE, 2005, p. 260).

Suporte adicional para assumir silent nouns como uma fronteira sintática na distribuição do morfema de plural no DP é fornecido pela posição de um morfema nulo de gênero feminino no russo (6) e também por um morfema nulo de número no árabe libanês (7), que dividem seus DPs em dois domínios para concordância nominal (PESETSKY, 2013). ${ }^{17}$ Nessa divisão, adjetivos altos no russo (6) são opcionalmente flexionados no feminino, enquanto adjetivos baixos são flexionados no masculino, quando nomes que indicam profissão se referem a mulher. No árabe (7), adjetivos altos ficam no singular, enquanto adjetivos baixos são opcionalmente flexionados no plural, quando a sentença contém um numeral maior que dez. Esse padrão espelha aquele apresentado no russo para gênero, de acordo com Pesetsky (2013).

“U nas byl-a očen' xoroš-aja zubn-oj vrač-ъ...

by us COP-PST.F.SG very good-F.NOM.SG dental-M.NOM.SG doctor-NOM.SG

'We had a very good (female) dentist."' (PESETSKY, 2013, p. 38).

"[tleetiin walad kesleen-Ø mnazzam-iin] Htajj-u

thirty child.SG lazy-SG organized-PL complained-PL

'Thirty organized lazy children complained (e.g., about their grades).'"

(PESETSKY, 2013, p. 47).

Em suma, de modo semelhante aos numerais cardinais, silent nouns funcionam como uma fronteira para a distribuição de traços de número no DP, um padrão encontrado nas línguas do mundo.

${ }^{16}$ Conforme observação de Charlotte Galves (UNICAMP), em comunicação pessoal, não há liaison na pronúncia de 'les une', em (5b). Esse fato contribui para confirmar a postulação de uma fronteira (um silent noun) entre 'les' e 'une' que, na fonologia, chega a produzir uma barreira, impedindo a liaison entre o 's' final de 'les' e a vogal inicial de 'une'.

${ }^{17}$ Agradeço a David Pesetsky (MIT) por ter despertado minha atenção para a proposta referente aos morfemas nulos. 


\section{FRONTEIRAS SINTÁTICAS NA DISTRIBUIÇÃO DO MORFEMA DE PLURAL EM PB NÃO PADRÃO}

Nesta seção, serão testadas, em PB não padrão, as predições apresentadas sobre valoração de traços de número e sua relação com a posição de cardinais e de silent nouns, no domínio do DP.

\subsection{Cardinais}

A valoração de traços de número em (1a), exemplo repetido abaixo, é descrita em (8) e resumida na Tabela 1.

a. Os (dois) outro carro branco

(8) a. $\mathrm{D}, \mathrm{A}_{\mathrm{s}}$ e $\mathrm{N}$ têm traços de número lexicalmente não interpretáveis e não valorados: $u \mathrm{~F}[\mathrm{]}$;

b. Num tem traços de número lexicalmente interpretáveis e valorados: $i \mathrm{~F}$ val;

c. D sonda Num e passa a ter seu traço de número valorado: $u \mathrm{~F}$ val;

d. $\mathrm{A}_{\mathrm{s}}$ e $\mathrm{N}$ estão sob o domínio de c-comando ${ }^{18}$ de NumP e constituem uma cadeia na qual compartilham os mesmos traços não valorados de número;

e. O A mais alto sonda Num e passa a ter seus traços de número valorados: $u \mathrm{~F}$ val;

f. Como consequência de estarem em uma cadeia com o $\mathrm{A}$ mais alto, $\mathrm{N}$ e o $\mathrm{A}$ mais baixo passam a ter seus traços de número valorados também: $u \mathrm{~F} v a l$.

Adiante (Tabela 1), encontra-se o resultado da combinação de traços de número em (1a):

Tabela 1: Traços de número antes e depois da realização de concordância

\begin{tabular}{llcc}
\hline & & $\begin{array}{c}\text { Traços de número antes da } \\
\text { concordância }\end{array}$ & $\begin{array}{c}\text { Traços de número depois da } \\
\text { concordância }\end{array}$ \\
\hline $\mathrm{D}$ & 'Os' & $u \mathrm{~F}[]$ & $u \mathrm{~F}$ val \\
Num & '(dois)' & $i \mathrm{~F} \mathrm{val}$ & $i \mathrm{~F}$ val \\
A alto & 'outro' & $u \mathrm{~F}[]$ & $u \mathrm{~F}$ val \\
A baixo & 'branco' & $u \mathrm{~F} \mathrm{[]}$ & $u \mathrm{~F}$ val \\
$\mathrm{N}$ & 'carro' & $u \mathrm{~F} \mathrm{[]}$ & $u \mathrm{~F}$ val \\
\hline
\end{tabular}

Fonte: Elaborado pelo autor.

18 Segundo Norris (2014, p. 104-105), “adjectival heads search their c-command domains for suitable goals as normal. Upon finding nothing to Agree with, the search continues upward, i.e., the probe search for a c-commanding goal rather than a ccommanded goal". 
Além disso, os diagramas (9a) e (9b) mostram a distribuição do morfema de plural nos sintagmas localizados na estrutura interna do DP, em (1a) e (1b), respectivamente.

Figura 1: Distribuição do morfema de plural no DP

(9) a.

Para (1a): Os (dois) outro carro branco

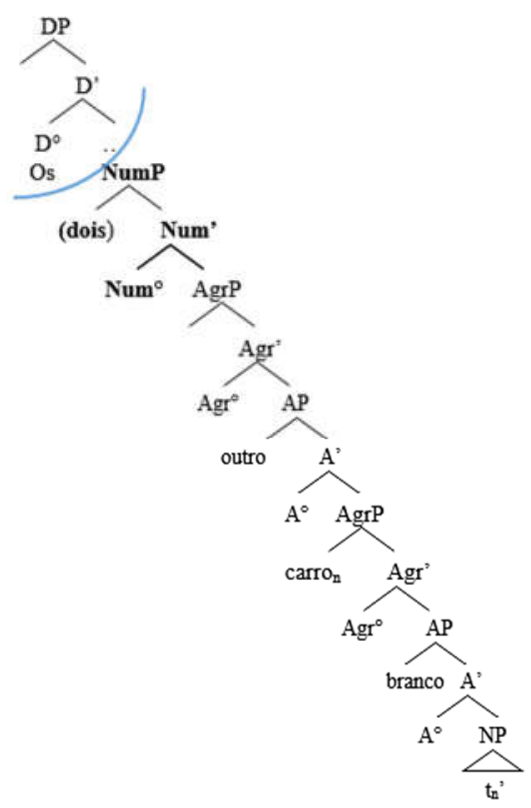

(9) b.

Para (1b): Os outros (dois) carro branco

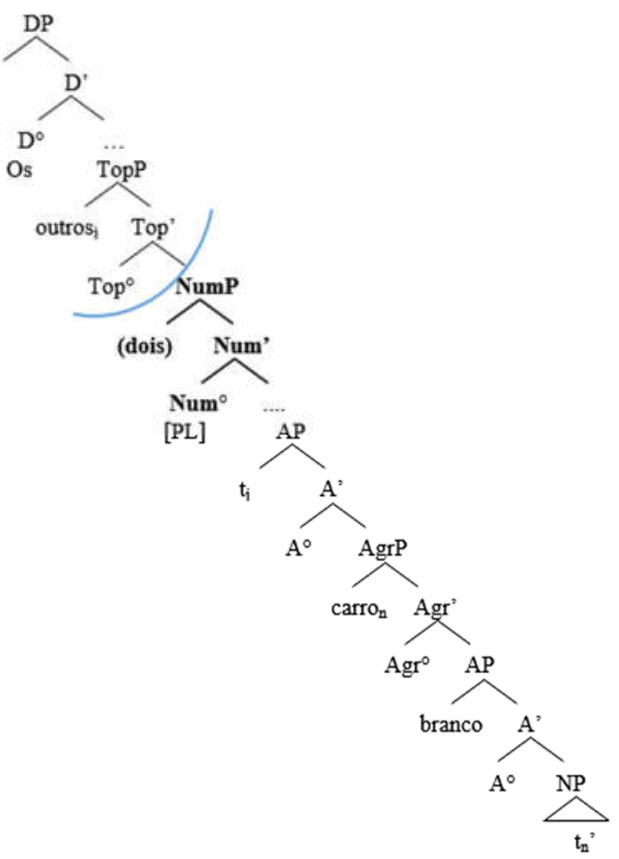

Fonte: (Elaborado pelo autor)

Antes de prosseguir com a análise da valoração de traços, é importante elaborar algumas considerações sobre as estruturas em (9a) e (9b), que se baseiam na proposta de Cinque (2005). Nessa proposta, cardinais são inseridos por merge em NumP, e a ordem universal das projeções funcionais internas ao DP é [DP NumP AP NP]. Outras ordens lineares possíveis são explicadas por movimento do NP como um XP para o especificador de posições AgrP, que são inseridas por merge com cada projeção funcional na estrutura do DP. Por exemplo, em (9a), o NP 'carro' é alçado por cima do AP mais baixo 'branco' para Spec,AgrP, o que explica a posição pós-nominal do adjetivo 'branco', como observado em (1a).

Em contraste, (1b) apresenta a seguinte ordem [DP AP NumP NP], com um AP precedendo NumP. Essa ordem não é gerada a partir de movimento do NP. Cinque (2005, p. 381, tradução minha) explica que "nem movimento de núcleo nem movimento de um sintagma que não contenha o NP (explícito) é possível (exceto talvez por movimentos, relacionados a foco, de sintagmas para uma posição inicial no DP)"19.

19 "Neither head movement nor movement of a phrase not containing the (overt) NP is possible (except perhaps for focus-related movements of phrases to a DP-initial position)" (CINQUE, 2005, p. 381). 
Considerando isso, Giusti (1996, p. 121, tradução minha) mostra que: "no italiano, adjetivos pré-nominais são sempre tópicos [...] Sua natureza dada pode ser enfatizada pelo fronteamento para uma posição imediatamente mais baixa que o DP" ${ }^{20}$. Esse é o caso de (1b), que deriva do movimento do AP 'outro' para Spec,TopP, como observado em (9b). Nessa posição, que é mais alta que NumP, $\mathrm{o}$ adjetivo recebe marca de plural.

Depois de explicar a estrutura do DP apresentada em (9), podemos retomar a questão da distribuição da marca de plural nos sintagmas internos ao DP. Em (1a) e (1b), D, N e A se tornam valorados com traços de número plural via concordância com Num, mas nem todos esses constituintes são marcados com o morfema de plural. Como argumentado acima, com base em Danon (2011) e Norris (2014), isso se deve a uma restrição imposta pela posição de NumP na estrutura do DP. Essa posição funciona como uma fronteira em que sintagmas acima de NumP são marcados com o morfema de plural, enquanto sintagmas que estão sob seu domínio de c-comando não são marcados.

Essa hipótese é também confirmada por (10) e (11) abaixo. Esses dados contêm outros adjetivos, além de 'outro' (1), que podem preceder o numeral cardinal, tais como 'primeiro' (10) e 'único' (11). Em (10a) e (11a), o cardinal 'dois' está situado logo após D; como resultado, somente D recebe a marca de plural, enquanto os sintagmas à direita do cardinal não são marcados. Em (10b) e (11b), o cardinal 'dois' está situado logo após D e A ('primeiros' ou 'únicos'); como resultado, somente D e A recebem a marca de plural. Em contraste, (10c) e (11c) são agramaticais seja porque sintagmas situados à direita do cardinal estão marcados com o morfema de plural, quando não deveriam estar, seja porque sintagmas situados à esquerda do cardinal não estão marcados com o morfema de plural, quando deveriam estar. As estruturas sintáticas de (10a, 11a) e de (10b, 11b) podem ser visualizadas em (12a) e (12b), respectivamente.

(10) a. Os (dois) primeiro carro branco

b. Os primeiros (dois) carro branco

c. *Os primeiro (dois) carros branco

(11) a. Os (dois) único carro branco

b. Os únicos (dois) carro branco

c. *O únicos (dois) carro brancos

20 "In Italian, prenominal descriptive adjectives are always topic [...] Their given nature can be emphasized by fronting into a position immediately lower than DP." (GIUSTI, 1996, p. 121). 
Figura 2: Cardinais como fronteira sintática na distribuição do morfema de plural no DP (12)

a. Para (10a) e (11a)

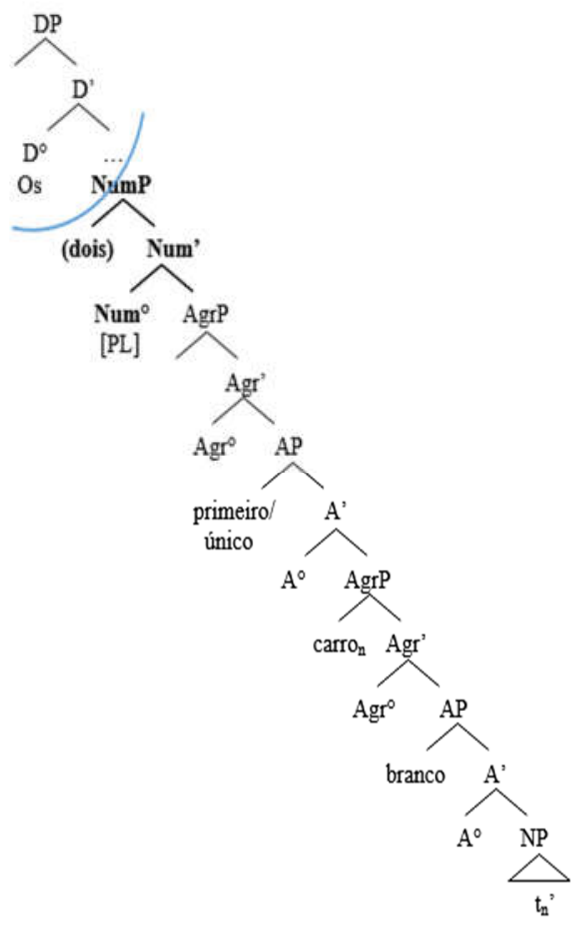

b. Para (10b) e (11b)

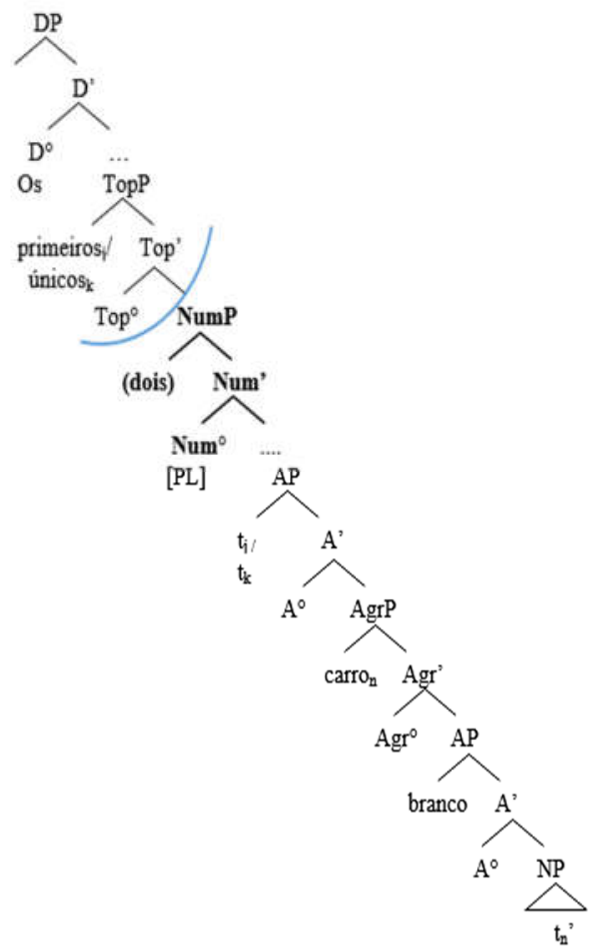

Fonte: Elaborado pelo autor.

Além disso, observa-se que a proposta referente aos cardinais como fronteira para a distribuição do morfema de plural nos sintagmas internos ao DP também contempla outras estruturas nominais no PB, como aquelas com possessivos e nomes nus.

No que diz respeito aos possessivos, como observado em (13a), o artigo 'os' e o possessivo 'meus', que precedem a posição do cardinal numeral, são marcados com o morfema de plural, enquanto o nome 'carro' e o adjetivo 'branco', que sucedem o cardinal, são não marcados.

a. os meus (dois) carro branco

Em posição pré-nominal, o possessivo precede cardinais (13a). Por essa razão, ele é sempre marcado com o morfema de plural, em DPs plurais. Com efeito, o artigo definido pode não receber a marca de plural (13b), o que não ocorre com o possessivo (13c). Portanto, (13b) poderia aparentemente representar um problema para a análise que estou assumindo porque, como o artigo está localizado à esquerda de NumP, ele deveria receber marca de plural assim como o possessivo.

(13) b. o meus (dois) carro branco

c. *os meu (dois) carro branco 
Nesse respeito, tem sido observado que, quando o artigo definido coocorre com possessivos pré-nominais, "o artigo definido [...] não é um marcador de definitude, mas apenas um expletivo" (COSTA; FIGUEIREDO SILVA, 2006, p. 40, tradução minha) $)^{21}$. Segundo essa visão, sendo um determinante expletivo, o artigo definido pode ser apagado ou ocorrer sem marcação de plural.

Uma análise desses fatos, apresentada em Pereira (2016b), é que o artigo definido, quando coocorre com possessivos pré-nominais, forma, com o possessivo pré-nominal, um único sintagma (DP) em que o artigo é o especificador (Spec,DP), enquanto o possessivo é o núcleo (D). A evidência mais forte para essa análise é a adjacência entre o artigo e o possessivo, como já se observou na literatura (CASTRO, 2001, p. 611). Por exemplo, em (13d), o cardinal não intervém entre o artigo e o possessivo. O mesmo se dá com o adjetivo, em (13e).
d. *os dois meu carro branco
e. *os único meu carro branco

Como o artigo definido e o possessivo prenominal formam juntos um único sintagma, o especificador (o artigo) é marcado opcionalmente com o morfema de plural, enquanto o núcleo (o possessivo) é marcado obrigatoriamente. Em vista desses fatos, a predição segundo a qual NumP divide a estrutura interna do DP em dois domínios se aplica: o DP (contendo o artigo e o possessivo) está acima de NumP, como mostrado em (14), o que faz com que ele receba a marca de plural. $\mathrm{O}$ fato de o artigo poder ser marcado opcionalmente não causa qualquer problema para esta predição, porque ele está dentro de um sintagma cujo núcleo já está marcado.

21 "the definite article [...] is not the marker of definiteness, and is just an expletive" (COSTA; FIGUEIREDO SILVA, 2006, p. 40). 
Figura 3: Cardinais como fronteira sintática na distribuição do morfema de

(14) Para (13a) e (13b) plural em estruturas com possessivos

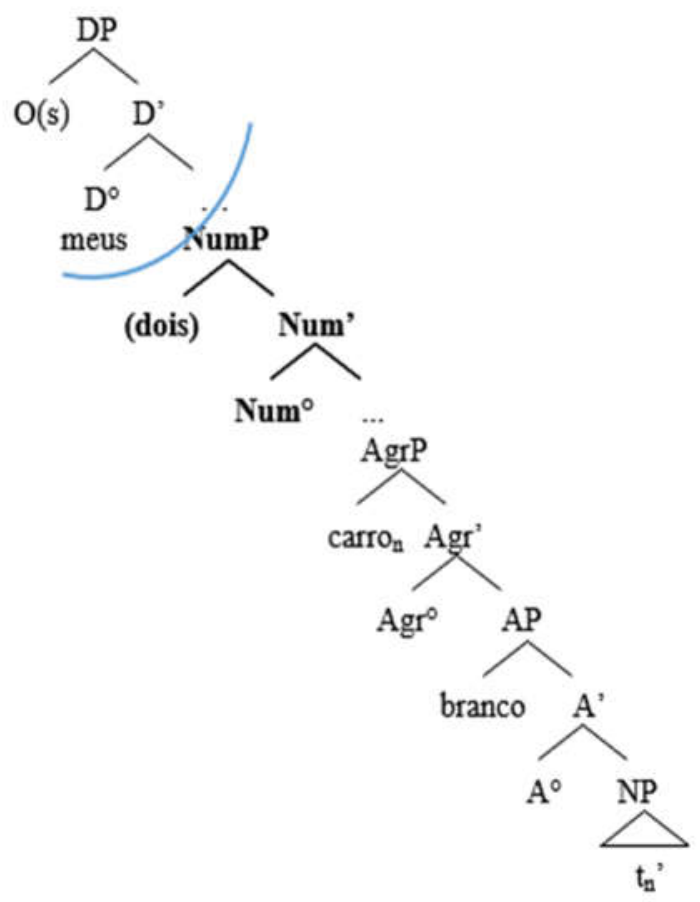

Fonte: Adaptado de Pereira (2016b)

No que diz respeito a nomes nus, em análises vigentes, o nome 'criança' em (15a) é conhecido como "singular nu" (bare singular) (MÜLLER, 2002; MUNN; SCHMITT, 2005, etc.).

(15) a. Vi criança brincando no jardim.

a'. Vi [NumP $\left.(\text { cinco })_{\mathrm{PL}}\right]$ criança brincando no jardim.

b". Vi [NumP $\left.(u m a)_{S G}\right]$ criança brincando no jardim.

Em contraste, na abordagem assumida aqui, a noção de "singular" nu não se aplica ao nome 'criança' em (15a), quando apresenta interpretação plural, porque, apesar de sua aparência morfológica de singular, o nome é valorado com traços de plural, via concordância com Num [PL]. Em outras palavras, mesmo que um cardinal não esteja explícito na sentença, a estrutura se abre à inserção de um cardinal e, portanto, NumP se realiza de forma nula em (15a). Dessa forma, o cardinal 'cinco' em (15a') é aleatório, servindo apenas para ilustrar que a posição de NumP existe em (15a) e pode ser preenchida.

Além disso, porque o nome está à direita de NumP (15a'), ele não é marcado com o morfema de plural '-s'. Com efeito, uma interpretação comum veiculada por (15a) é aquela segundo qual há crianças brincando no jardim. Isso é confirmado pelo fato de que 'criança' pode ser retomado com o pronome no plural 'elas'. Por essas razões, 'criança' em (15a) não deveria ser classificado como "singular" nu, nessa interpretação que acaba de ser explicada.

A favor disso, refiro-me à discussão apresentada por Augusto et al. (2006). Esses autores citam Deprez (2005, p. 867, tradução minha) que defende que, “em 
línguas +PL, NumP deve ser projetado sistematicamente, para nomes contáveis no mínimo - isto é, mesmo quando o sintagma nominal é singular, sem qualquer morfologia explícita de 'plural' ou 'singular' _"22. Com base nessa observação, Augusto et al. (2006, p. 265) concluem que, diferentemente de nomes de massa ${ }^{23}$, singulares nus são especificados para número. Assim, se Num é especificado para plural, plurais nus são gerados; mas, se Num é não especificado para plural, singulares nus são gerados.

Em outras palavras, se Num é valorado com traços de plural, nomes nus se tornam valorados com plural via Agree, como em (15a'), resultando na geração de plural nu em (15a). Diferentemente, se Num é valorado com traços de singular, nomes nus são valorados com singular via Agree, como em (15a"), resultando na geração de singular nu em (15a). Com efeito, uma outra interpretação possível de (15a) é aquela segundo qual há uma criança (qualquer) brincando no jardim. Nesse caso, 'criança' pode ser retomado com o pronome no singular 'ela'.

Em suma, vários exemplos evidenciam que NumP é uma fronteira na distribuição do morfema de plural, na estrutura interna do DP em PB.

\subsection{Silent nouns}

Há certas configurações nominais, como em (16a), que não permitem a ocorrência de cardinais. Se marginalmente o fazem, como em (16b), o conteúdo proposicional veiculado pela sentença não é o mesmo que aquele veiculado em (16a). Em (16a), entende-se "umas meia hora" como "cerca de meia hora", enquanto em (16b), entende-se "umas duas meia hora" como dois blocos de meia hora. Em configurações nominais que não projetam NumP, assumo que existe um silent noun, nos moldes da descrição de Kayne (2005), que atua como uma fronteira na distribuição do morfema de plural no DP.

(16) a. Levou umas $[X P$ HOUR PL of $]$ meia hora pra (cólica) passar.

b. ??Levou umas [Numpduas] meia hora pra (cólica) passar.

Em observação à hipótese de Kayne (2005) sobre silent nouns e a evidências fornecidas no inglês (4), italiano (5a) e francês (5b), assumo que (16a) apresenta um silent noun, em vez de NumP, com traços de plural. Com efeito, o francês $(5 b)$ revela um padrão quase idêntico ao apresentado no PB (16a), porque um determinante no plural (les no francês e umas no PB) é seguido por sintagmas no singular (une heure no francês e meia hora no PB).

\footnotetext{
22 "In + PL languages, NumP must systematically project, for count nouns at least - that is, even when the noun phrase is singular with no apparent overt 'plural' or 'singular' morphology -" (DEPREZ, 2005, p. 867).

${ }^{23}$ Em certos casos, mesmo nomes de massa são compatíveis com cardinais, como em "Comprei três água" ou "Usei dois açúcar no café". Nessas estruturas, fica implícito, após o cardinal, um nome indicador de continente, como 'garrafa de' ou 'sachê de', respectivamente.
} 
Esses fatos podem ser explicados pela presença de uma categoria nula HOUR com traços de plural. Logo, em (16a), porque 'umas' precede HOUR, é marcado com '-s'. A estrutura em (16a) também é comum com outros nomes de

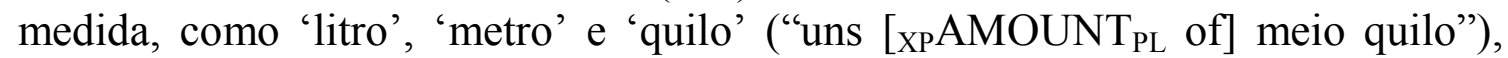
que também admitem um silent noun no plural do tipo AMOUNT.

Além disso, esse silent noun $^{24}$ no plural é seguido por uma preposição, como 'of' em (4c), e resultaria em algo semelhante a "uns (pesos de) meio quilo", no caso de AMOUNT (of), ou "umas (demoras de) meia hora", no caso de HOUR (of $)^{25}$. Isso permite que sintagmas encaixados na posição de complemento da preposição estejam no singular, como 'meia hora', em (17), enquanto sintagmas à sua esquerda estejam no plural, como 'umas'.

\footnotetext{
${ }^{24}$ Fica em aberto, no momento, que rótulo essa categoria funcional [XP] deve ter e se ela é seguida por um PP, como em [XP[PP]].

${ }^{25}$ A inserção de 'pesos de', em 'uns meio quilo', e de 'horas de' ou 'demoras de', em 'umas meia hora', não tem como fim uma paráfrase, mas uma amostra de que tais estruturas permitem a presença de um nome com valor de quantidade (AMOUNT) ou de tempo (HOUR) entre o artigo indefinido e as expressões de medida com 'meio(a)'. Para uma paráfrase, com inserção nominal, algumas possibilidades seriam: "umas proximidades de meia hora" (cerca de meia hora) ou "uns aproximados de meio quilo" (cerca de meio quilo). Porém, nesse caso, tais nomes não explicitam as noções de AMOUNT ou de HOUR dos silent nouns pertinentes a cada caso. Além disso, não se pode perder de vista que o valor aproximativo é dado pelo artigo indefinido "um(a)" e não pela marca de plural. Por isso, discordo da afirmação de um parecerista segundo a qual "esse plural em 'uma' parece indicar 'quantidade aproximada"'. Ora, mesmo sem a marca de plural, tem-se valor aproximativo: "Levou uma meia hora pra dor passar". Discordo também de outra afirmação segundo a qual, em "umas boas meia hora", se 'boas' modificar um nome (silent noun), como em 'umas boas demoras de meia hora', "só teremos a leitura avaliativa de 'boas', como em 'bom homem'". Ora, não é essa a leitura veiculada na expressão, e outras questões estariam envolvidas na interpretação de 'boas', como a posição pré-nominal do adjetivo ('grande homem' x 'homem grande'). Assim, 'umas boas meia hora' não significa uma meia hora boa, mas cerca de meia hora (ou mais). Portanto, a discussão apontada desse exemplo não procede como contraargumento. Ao contrário, "umas boas meia hora" vem a corroborar a proposta dos silent nouns como fronteira sintática na distribuição do morfema de plural no DP, visto que 'umas' e 'boas' estão marcados com o plural, por estarem à esquerda do silent noun, enquanto 'meia' e 'hora' não recebem tal marca, por estarem à direita: "umas boas [HOUR of] meia hora".
} 
Figura 4: Silent nouns como fronteira sintática na distribuição do morfema de plural no DP

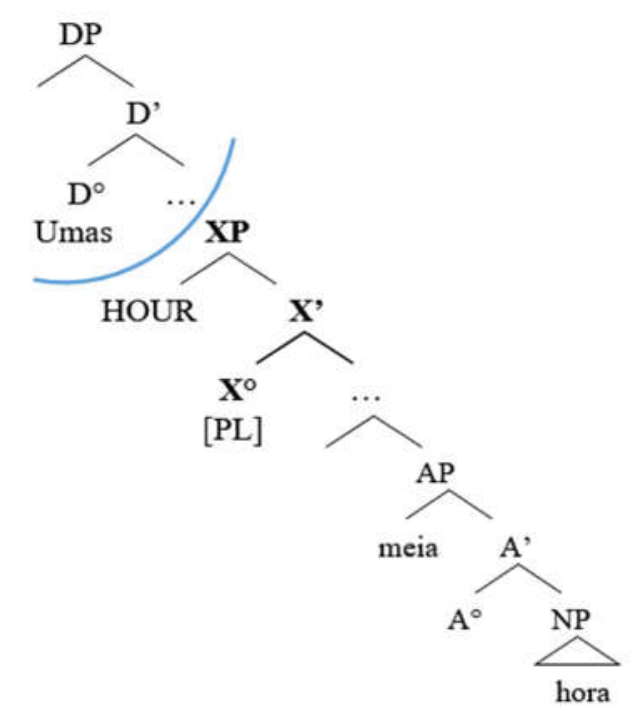

Fonte: (Elaborado pelo autor)

Resta destacar ainda que (18) fornece evidência independente para a presença de uma categoria funcional nula HOUR com traços de plural, porque, em (18), o artigo 'umas' está flexionado não somente no plural mas também no feminino - que é o gênero de 'hora' -, embora preceda a palavra 'quinze', que se refere a 'minutos', uma palavra masculina.

(18) Eu cheguei ... era umas $\left[\mathrm{XPHOUR}_{\mathrm{FEM.PL}}\right.$ of $]$ quinze pras sete (Passageiro entrevistado pelo Jornal Hoje, Aeroporto de Congonhas, 18 de julho de 2016).

Portanto, categorias funcionais nulas desempenham o papel de fronteira sintática na distribuição do morfema de plural no DP.

\section{EVIDÊNCIAS PROVINDAS DE DADOS DE PB DIALETAL}

Adiante, serão analisados exemplos recorrentes no dialeto mineiro que evidenciam as propostas acima discutidas sobre cardinais e silent nouns como fronteiras na distribuição do morfema de plural, na estrutura do DP.

Por exemplo, em (19a), 'ques' é um determinante, como mencionado por Nunes (2007), e precede NumP, como observado em (20b). Por essa razão, 'que' é marcado com o morfema de plural, enquanto os constituintes à direita de NumP - 'coisa' e 'sofrível' - são não marcados. 
(19) a. Ques (duas) coisa sofrível! ${ }^{26}$

b. Ques (duas) coisa sofrível (que é essas)!

Em Pereira (2016a), propus que a forma flexionada 'ques' [kis] resulta de analogia com 'quais', que é a forma flexionada de 'qual', também pronunciada como [kis] em PB não padrão. Ademais, propus que a estrutura sintática de (19a, b) é representada em (20a). Nesse diagrama, o DP ao qual 'ques' pertence é predicado de uma small clause (SC), que está dentro de uma sentença exclamativa (19b). Esse DP alça da posição de predicado da small clause, para o domínio do CP, de modo a checar força ilocucionária, como observado em (20a).

Figura 5: Cardinais como fronteira sintática na distribuição do morfema de plural em estruturas com 'ques'

(20) a. Para o CP em (19)

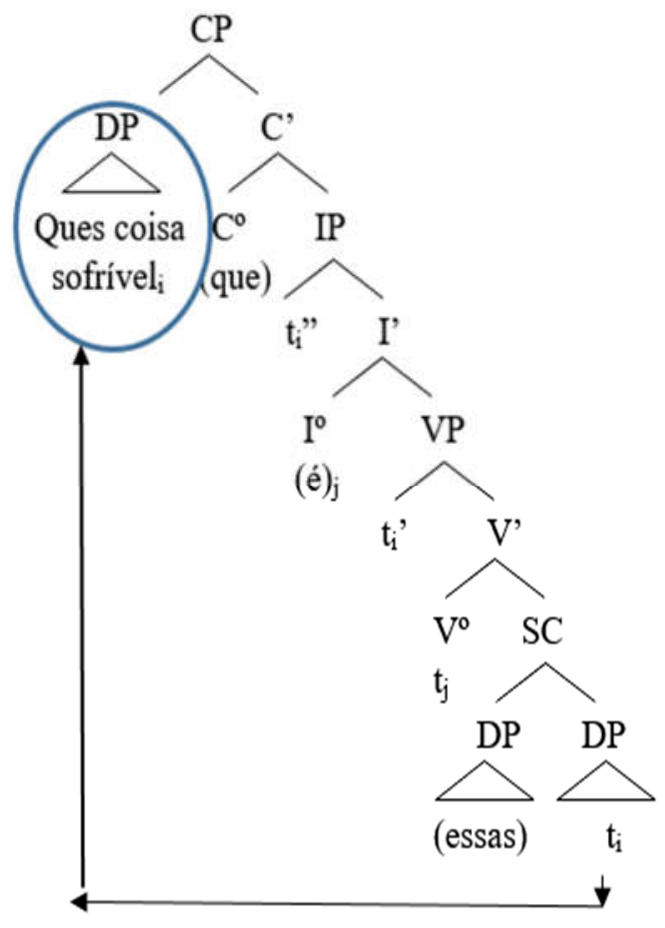

(20) b. Para o DP em (19)

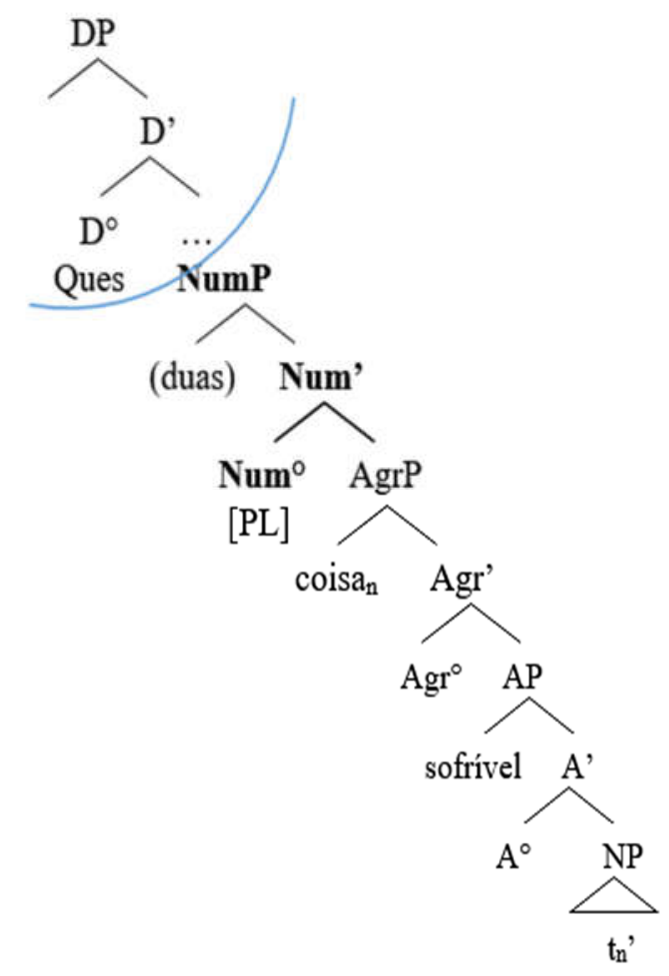

Fonte: Adaptado de Pereira (2016a)

Entretanto, cardinais nem sempre são compatíveis com itens wh (21b). Por exemplo, como numerais cardinais não podem aparecer em (21a), não há evidência da presença de NumP nesse DP.

a. Quantos que custa esse?

b. *Quantos três real que custa esse?

${ }^{26}$ Esse exemplo pode ser ouvido no minuto 21'20" de um vídeo do Canal High Torque, Belo Horizonte (MG). Disponível em:

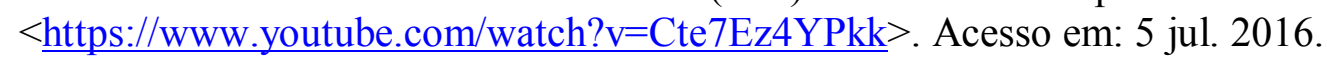


Como não há NumP na estrutura, não pode ser essa categoria que funciona como fronteira na distribuição do morfema de plural, em (21a). Uma hipótese inicial poderia ser que o próprio quantificador 'quantos' desempenha esse papel e entra na derivação com traços de número valorados. No entanto, 'quantos' é um determinante $w h$ (D) como 'quais' e 'que'. Como defendido acima, traços de número no PB são não valorados e não interpretáveis em D. Logo, em (21a), como NumP não é projetado, 'quantos' sonda outra categoria para valoração de traços de número, qual seja, um silent noun.

Essa hipótese considera a proposta sobre silent nouns, devido ao fato de que, em (21a), 'quantos' projeta um silent noun do tipo AMOUNT (of), que resultaria em algo semelhante a "quantos (valores de) real". Portanto, porque 'quantos' precede uma projeção nula com traços de plural, 'quantos' é marcado com '-s', como se observa em (22b).

Figura 6: Silent nouns como fronteira sintática na distribuição do morfema de plural em estruturas com 'quantos'

(22) a. Para o CP em (21a)

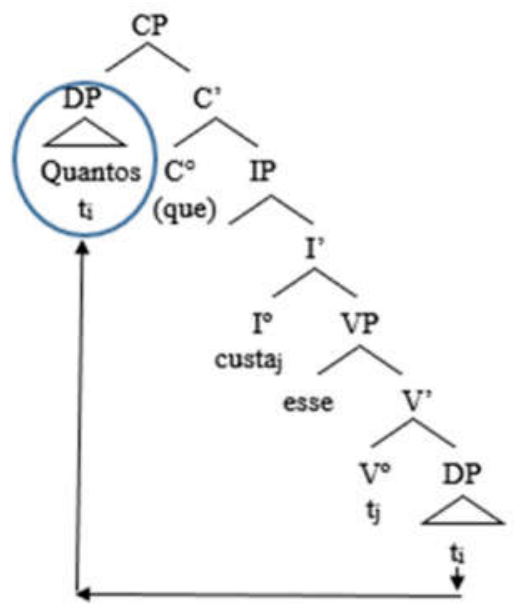

Fonte: (Elaborado pelo autor)

(22) b. Para o DP em (21a)

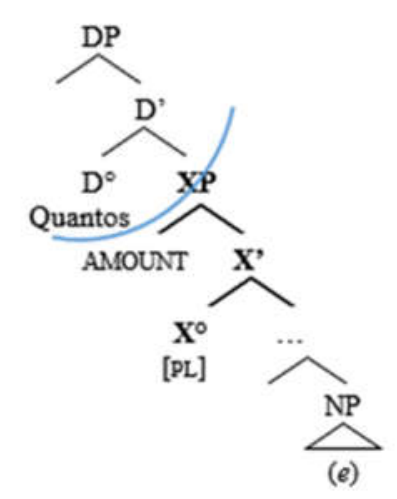

Um outro aspecto a ser mencionado a respeito de (21a) é que 'quantos' veicula a leitura não contável 'how much' (como em 'Quanto custa?'), embora a leitura contável 'how many' também seja possível (como em 'Quantos reais custa?'), quando se considera uma estrutura na qual um nome, como 'real', esteja elíptico: "Quantos real". Desse modo, pode-se propor que essas duas leituras estejam disponíveis na mesma estrutura (21a), porque a palavra 'quanto' não contável ('how much') está sendo pronunciada como uma forma flexionada, com o morfema de plural '-s'. Esta forma resulta de analogia com a forma contável e flexionada 'quantos' ('how many').

Essa analogia é semelhante àquela que ocorre entre 'ques' e 'quais', como mencionado acima. Um outro aspecto semelhante de estruturas com 'ques' $\mathrm{e}$ 'quantos' é que o DP ao qual esses determinantes $w h$ pertencem alçam para o domínio do CP em exclamativas (20a) e em interrogativas (22a). 
Em suma, ocorrências ${ }^{27}$ do dialeto falado em Minas Gerais mostram que cardinais e silent nouns funcionam como uma fronteira para a distribuição do morfema de plural na estrutura interna do DP.

\section{CONCLUSÃO}

Este artigo propõe uma análise para a distribuição do morfema de plural na estrutura interna do DP, em PB não padrão, com base principalmente na proposta de Danon (2011) e Norris (2014) sobre cardinais e na proposta de Kayne (2005) sobre silent nouns.

Nessa análise, a posição do numeral cardinal (NumP) divide o DP em dois domínios, de modo que constituintes situados acima dessa posição são marcados com o morfema de plural, enquanto constituintes que estão abaixo dessa posição são não marcados. Em certas estruturas que não projetam NumP, assumo que um silent noun funciona, em vez de cardinais, como fronteira para distribuição do morfema de plural. Esses padrões dos quais o PB apresenta evidências robustas são atestados em outras línguas.

Em conclusão, ao contrário de propostas vigentes que argumentam a favor de um componente morfológico independente, a análise que se assume aqui revela que a distribuição do morfema de plural no DP, no PB, é determinada pela sintaxe, isto é, pela posição de cardinais e silent nouns na estrutura do DP.

\section{REFERÊNCIAS}

AUGUSTO, Marina R.; FERRARI NETO, José; CORRÊA, Letícia. Explorando o DP: a presença de NumP. Revista de Estudos da Linguagem. Belo Horizonte, n. 14 , v. 2, 2006, p. 245-275.

BLÜHDORN, Hardarik; SIMÕES, Luciene; SCHMALTZ, Márcia. Sintagmas nominais contáveis e não-contáveis no alemão e no português brasileiro. In: BATTAGLIA, Maria; NOMURA, Masa (Org.). Estudos linguísticos contrastivos em alemão e português. São Paulo: Annablume, 2008. p. 41-82.

CASTRO, Ana. Os possessivos em português europeu e português brasileiro: unidade e diversidade. In: ENCONTRO NACIONAL DA ASSOCIAÇÃO PORTUGUESA DE LINGUÍSTICA, 16., 2001, Lisboa. Actas... Lisboa: APL, 2001. p. 599-613.

${ }^{27}$ Está em andamento (PEREIRA, 2018) uma análise de outras ocorrências que trazem evidências para essa proposta, como a flexão de quantificador 'cada', em estruturas do tipo "cadas vestido horrível". 
CASTRO, Ana; PRATAS, Fernanda. Capeverdean DP-internal number agreement: additional arguments for a distributed morphology approach. In: COSTA, João; FIGUEIREDO SILVA, Maria C. (Org.). Studies on agreement. Amsterdam/Philadelphia: John Benjamins, 2006. p. 11-24.

CHOMSKY, Noam. Derivation by phase. In: KENSTOWICZ, Michael (Ed.). Ken Hale: a life in language. Cambridge, MA: The MIT Press. 2001. p. 1-52.

CINQUE, Guglielmo. Deriving Greenberg's Universal 20 and its exceptions. Linguistic Inquiry. Cambridge, MA, v. 36, n. 3, 2005, p. 315-332.

COSTA, João; FIGUEIREDO SILVA, Maria. Nominal and verbal agreement in Portuguese: an argument for distributed morphology. In: (Org.). Studies on agreement. Amsterdam/ Philadelphia: John Benjamins, 2006. p. 25-46.

DANON, Gabi. Agreement and DP-Internal Feature Distribution. Syntax, Haboken, NJ, v. 14, n. 4, 2011, p. 297-317.

DEPREZ, Viviane. Morphological number, semantic number and bare nouns. Lingua, Amsterdam, n. 115, 2005, p. 857-883.

KAYNE, Richard. Movement and Silence. Oxford; New York: Oxford University Press, 2005.

MÜLLER, Ana. Genericity and denotation of common nouns in Brazilian Portuguese. DELTA. São Paulo, v. 18, n. 2, 2002, p. 287-308.

MUNN, Alan; SCHMITT, Cristina. Number and indefinites. Lingua, Amsterdam, n. 115,2005 , p. 821-855.

NORRIS, Mark. A theory of nominal concord. Santa Cruz, California: 2014. 276 f. Tese de Doutorado, University of California, Santa Cruz.

NUNES, Jairo. Triangulismos e a sintaxe do português brasileiro. In: CASTILHO, Ataliba; KATO, Mary (Org.). Descrição, história e aquisição do português brasileiro. Campinas: Pontes, 2007. p. 25-34.

PEREIRA, Bruna K. Exclamatives and interrogatives with 'ques': the CP/DP hierarchy and the plural marking in Brazilian Portuguese. Signótica. Goiânia, v. 28, n. 2, 2016a , p. 581-611.

PEREIRA, Bruna K. Feature interpretability and the positions of $2^{\text {nd }}$ person possessives in dialectal Brazilian Portuguese. Filologia e Linguística Portuguesa. São Paulo, v. 18, n. 2, 2016b, p. 199-229.

PESETSKY, David. Russian case morphology and the syntactic categories. Cambridge: MIT Press, 2013. 
PESETSKY, David; TORREGO, Esther Torrego. The syntax of valuation and interpretability of features. In: KARIMI, Simin et al. (Ed.). Phrasal and clausal architecture. Amsterdam: John Benjamins, 2007. p. 262-294.

Bruna Karla Pereira BrunaUFMG@yahoo.com.br

Recebido em: 15 jan. 2018 Aceito em: 15 abr. 2018 Publicado em: 1 out. 2018 